Mitja Skubic

\title{
LE PASSÉ SIMPLE ET LE PASSÉ COMPOSE DANS LA LANGUE DES TROUBADOURS
}

1. - E. Bourciez constate ${ }^{1}$ que l'ancien français et l'ancien provençal se servent assez librement du passé composé et du passé simple. Cela signifie que les deux formes verbales ne sont pas, ou mieux, ne sont plus différenciées par leur valeur, comme elles l'étaient sans doute à la naissance de la forme périphrastique ${ }^{2}$.

Une analyse plus détaillée de l'emploi des deux passés, simple et composé, dans la poésie lyrique en ancien provençal montre toutefois que les deux formes verbales possèdent chacune une valeur propre et que, par conséquent, elles ne sont pas interchangeables.

2. - Nous trouvons le passé composé surtout dans les cas où l'on exprime l'état présent, résultant d'une action passée: la valeur du passé composé est donc celle du parfait. Par contre, nous trouvons le passé simple quand c'est l'action verbale pure que l'on a voulu exprimer; la valeur du passé simple est donc celle de l'aoriste.

Ici, nous avons donc à faire avec l'opposition aoriste : parfait qui est née au moment où l'on ajouta, dans le latin parlé, au perfectum simple du latin littéraire un parfait périphrastique.

3. - Il est le plus facile de constater la valeur du parfait avec des verbes qui, par leur nature même, expriment l'état et par conséquent dans la majeure partie des langues romanes exigent, presque tous, pour les temps composés, l'auxiliaire être; on trouve des exemples comme:

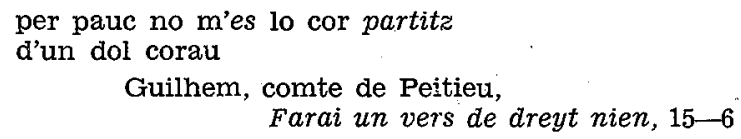

${ }^{1}$ E. Bourciez: Eléments de linguistique romane, 4ème éd., Paris, 1946, par. $318 \mathrm{c}$ : Enfin, pour l'expression même du passé proprement dit, il y avait hésitation entre le parfait simple il chanta et les deux périphrases il a chanté, il ot chanté. En a. français, ces diverses formes peuvent se substituer les unes aux autres, et se mélangent très librement entre elles, de même qu'avec le présent: Ço sent Rollanz que s'espee li tolt, ovrit les uelz, si li a dit (Roll, 2284); li quens ne s'oublia mie, ains ot mandé ses homes (Aucassin 8,2 ); il mist le main a l'espee, si comence a ferir ( Ib. 10, 24). On trouve au Midi des phrases analogues: Pres lo entre sos bratz, si l'a baizat (Gir. de Ross 8101); cependant elles sont rares chez les Troubadours, dont la langue était plus artistique.

2 Pour nous, les deux termes de passé simple et de passé composé sont à prendre à la lettre, c'est-à-dire qu'ils concernent exclusivement la forme et nullement la valeur. 
Ai, mala fos reis Lozoics

que fai los mans e los prezics, per quel dols m'es el cor intratz

Marcambru,

A la fontana del vergier, $27-8$

Lanquand li iorn son lonc en mai,

m'es bels douz chans d'auzels de lonh,

e quand me sui partitz de lai,

remembra'm d'un'amor de lonh

Jaufre Rudel,

Lanquand li iorn son lonc en mai, 1-4

cazutz sui en mala merce,

et ai ben fait co'I fols en pon

Bernart de Ventadorn,

Quant vey la lauzeto, mover, $37-8$

per mi'us o dic, que mals m'en es vengutz, qu'enganat m'a la bela de mal aire

Id.,

Be m'an perdut, lai enves Ventadorn, 34-5

Pos tornatz sui en Proensa

et $a b$ ma domna:m sap bo,

ben dei far gaia chanso

Peire Vidal,

Pos tornatz sui en Proensa, 1-3

Dolen e trist e ple de marrimen

son remasut li cortes soudadier

e'lh trobador e'lh joglar avinen

Bertran de Born,

Si tuit li dol eilh plor e'lh marrimen, 9-11

Ar es anatz sos gens cors amoros,

don es dolors e desconortz et ira

ib., $31-2$

L'auteur aura recours au pasé simple quand il voudra exprimer l'aoriste, l'action verbale" pure:

En Alvernhe, part Lemozi,

m'en aniey totz sols a tapi.

trobey la moler d'en Guari

e d'en Bernart;

saluderon me simplamentz

per sanh Launart

Guilhem, comte de Peitieu,

Farai un vers, pos mi somelh, 13-8

e s'aissi es perduz lo bos talanz

que m'aviaz, quant nos partim amdui

Uc Catola,

Non posc mudar, bels amics, 5-6 
Cel sui que no soana

lo bei que Deus li fai, qu'en aquela setmana, quant eu parti de lai mi dis en razo plana que mos chantars li plai

Bernart de Ventadorn,

Quan la douss'aura venta, 51-6

e'm dis: mmorgue; quar venguis?"

Lo: Monge de Montaudon, L'autrier fuy en paradis, 6

4. - Avec des verbes transitifs et, en général, avec la grande majorité des verbes qui demandent dans la conjugaison des temps composés l'auxiliaire avoir, nous constatons la même opposition entre les deux valeurs exprimées au moyen des deux formes verbales. Certes, là où le passé simple est en train de disparaître, restant seulement le privilège de la langue littéraire, mais où il est inconnu de la langue parlée - et c'est-justement le cas de presque toutes les langues romanes d'aujourd'hui -, cette opposition n'est plus constatable. Le provençal des temps des troubadours, par contre, connaît les deux formes prétéritales et s'en sert avec une distinction très nette: comme celle avec l'auxiliaire être, la périphrase avec avoir exprime, elle aussi, l'état dans le présent, et a donc la valeur du parfait, tandis que la forme simple exprime I'action verbale pure, l'aoriste. On trouvera:

quar senes lieys non puesc viure,

tant ai pres de s'amor gran fam

Guilhem, comte de Peitieu, Farai chansoneta nueva, 11-2

Er'auziretz qu'ai respondut

Id., Farai un vers, pos mi somelh, 25

So dis n'Agnes a n'Ermessen:

strobat avem qu'anam queren ....

$$
\text { Ib., } 31-2
$$

Ben voill que sapchon li pluzor

un verset de bona color

qu'eu ai trait de mon obrador

Id., Ben voill que sapchon li pluzor, 1-3

Compaigno, non posc mudar qu'eu no m'estrei

de novellas qu'ai auzidas e que vei

Id., Compaigno, non posc mudar tec., 1-2

I'ot ai guerpit quant amar soill

Id., Pos de chantar m'es pres talens, 33 
ai, las! tan suavet m'aucis quan de s'amor me fetz sembian, que tornat m'a en tal deves que nuill'autra non vuelh vezer

Cercamon,

Quant l'aura doussa s'amarzis, 45-8

e pus lo temps si melhura, elh s'an lur ioya conquiza. Ara perdon l'alegragge pel frey e per la gilada; ma ieu ai pres tal usatge: totz iorns chant...

Marcambru,

Lanquan fuelhon li boscatge, 6-11

ia no'm deu esser escura d'aquo don tan l'ay enquiza.

Be'm tengratz per folhatura, si b'm fai e mielhs m'ahura, s ieu ia m'en planc quar l'ay viza

$$
\text { ib., } 41-5
$$

cum nos a fait per sa dousor

lo seingnorius celestiaus

$$
\text { Id., Pax in nomine Domini, 4-5 }
$$

S'al prim yos $f i$ ren que no'us fos a grat, si m'aiut Deus, ben o ai car comprat

\section{Uc Catola,}

Non pose mudar, bels amics, 8-9

Des exemples analogues se trouvent, nombreux, aussi dans les vers de Bernart de Ventadorn:

Be m'an perdut, lai enves Ventadorn, tuit mei amic, pos ma domna no m'ama

Be m'an perdut ecc., 1-2

per mi 'us o dic, que mals m'en es vengutz, qu'enganat m'a la bela de mal aire

$$
\text { ib., } 34-5
$$

vas cui e sui aclis, en cui $a i$ mes m'ententa e mon coratge assis

Quan la douss'aura venta, $6-8$

Miralhs, pos me mirei en te m'an mort li sospir de preon, qu'aissi'm perdei cum perdet se lo beis Narcissus en la fon

Quant vei la lauzeta mover, 21-4

mas be n'ai fiansa, que sivals eu n'ai conquiza la bela semblansa

Tant ai mo cor ple de ioya, $26-8$ 
Le passé simple et le passé composé dans la langue des troubadours

De l'oeuvre de Bertran de Born ón pourrait citer:

Dompna, puois de mi no'us cal e partit m'avetz de vos senes totas ochaisos, non sai...

... e si del semblan

non trob dompna a mon talan

que valha vos qu'ai perduda,

ia mais non vuolh aver druda

Dompna, puois de mi no'us cal, 1 -...10

E puois lo reis e'l coms Richartz

m'an perdonat lor mals talans

ja mais...

no laissarai hort

Ges de far sirventes no'm tartz, $10-\ldots 5$

5. - Il est inutile de citer les cas avec le passé simple dont la valeur est celle de l'aoriste. On la constate, cette valeur, pour toutes les actions dans un récit. En dehors du récit, il suffira de mentionner, en plus des cas déjà notés, l'usage qu'on trouve dans Peire Vidal:

et eu per lonc esperar

ai conquist ab gran doussor

lo bais que forsa d'amor

me fetz a mi dons emblar

Pos tornatz sui en Proensa, 50-3

$\mathrm{E}$ pos Deus vos fetz ses par

e'us det mi per servidor,

servirai vos...

ib., $59-61$

E s'eu sai ren dir ni faire,

ilh n'aia'l grat, que sciensa

m'a donat e conoissensa

per qu'eu sia gais chantaire

Ab l'alen tir vas me l'aire, 22-5

qu'ab bels semblans m'a mes en mortal pena

don ia ses leis no cre aver garensa

Seu fos.en cort on hom, 10-1

6. - Dans le cadre de l'opposition aoriste : parfait, il faut porter un intérêt tout particulier à des exemples comme à celui-ci de Peire Vidal:

Quant ai vestit mon blanc ausberc doblier e cenh lo bran que'm det en Gui l'autrier,

la terra crotia per aqui on eu vau

Drogoman, senher, s'eu agues bon destrier, 14-6 
Dans le passage cité, le passé composé exprime l'état dans le présent, l'état contemporain et parallèle à l'action représentée par le présent crotla. Ici, le passé composé n'a certainement pas la valeur d'une action toute récente: une determination, une locution adverbiale qui fixe l'action verbale à un passé récent, n'est vraiment pas imaginable. Et le passage cité montre justement qu'il ne s'agit pas d'une action - on la trouve dans que'm det en Gui l'autrier - mais d'un état.

Il est vrai, pourtant, que l'interprétation avec une action itérative serait possible: quant pourrait introduire une telle action, ayant aussi le sens de toutes les fois que; interprété ainsi, le verbe exprime une action d'aspect non ponctuel et le sens $d u$ verbe admettrait une locution adverbiale de temps: Quand j'ai mis, le matin,... mon blanc haubert ecc. Tout de même; nous croyons voir dans cet exemple le passé composé à valeur de parfait, mais ce sont justement de tels passages qui forment le pont entre la valeur de parfait. et celle de l'action non ponctuelle.

7. - Toute une série d'exemples analogues se trouve dans la poésie épique des troubadours. Nous trouvons dans l'épopée Girart de Rossilho:

Girartz jac en l'arvolt, no“i ac sirven

mas sa molher que'l sierve molt dossamen.

$\mathrm{Ab}$ tan veus Michael que a lui ven:

Dieus lo lhi a trames tot veramen.

Cel lhi portet un drap, denan lo'th ten

$$
\text { man. } 0 \text { vv. 7621-5 }
$$

Lo jorns es espasatz, e'l sers vengutz: Quan la nuhs fo vegnuda, l'escurs cazutz, adonc fo grans la noisa e lo tabust de monges, de canorgues, de clercs menutz. La reina au mostier en va pes nutz, e Girartz se levet, lai n'es vengutz

E quan sunt en la tor, cridunt: strait ! lautr'ant de fuc conmes lo reimarit. E Girarz s'esedet la u durmit, vit la clartat del fuc e ot lo crit. d'armes e de cheval son cors garnit (e ne furent mais catre a escharit) es venguz al portal e at l'ubrit e vit defors de lor tant elm brunit; per consence Milon lo. duc n'isit.

$$
\text { vv. } 6262-70
$$

Ces passages attestent les deux formes du passé et le présent en plus: le cas est donc exactement identique à celui cité par Bourciez. Et il est vrai, du moment qu'il s'agit d'un récit, que les passés composés nous laissent perplexes: lec actions verbales qui le constituent se succèdent l'une après. l'autre comme liées dans une chaîne, une action terminée, la suivante prend. sa place. C'est pourquoi on s'attendrait au passé simple, comme en le trouve.. régulièrement dans un roman, pour le récịt. 
Toutefois, il semble qu'il s'agisse moins d'une incohérence dans l'emploi des temps que d'une vaieur stylistique du passé composé, d'une valeur styJistique qui, pourtant, est entièrement basée sur la valeur grammaticale de la Lorme verbale: dans tous ces cas le passé composé a la valeur de parfait, d'un état dans le présent donc. La poésie épique s'en sert pour sa valeur stylistique, son emploi a l'avantage d'un effet tout particulier: le passé composé à valeur de parfait exige derrière lui une brève pause logique ${ }^{3}$ et par suite, interrompt le cours régulier de la narration. Ayant substitué dans la rapide, mais tranquille, régulière narration de venc al portal e l'ubrit e vit la forme composé à la forme simple, ou mieux, le parfait à l'aoriste, en es venguz al portal et at l'ubrit e vit, l'auteur a, par la forte césure après at l'ubrit, comme l'état dure encore quand l'action suivante sera réalisée, mis en relief l'action verbale qui suit.

Il est à noter que l'imparfait, dans le Roman de Girart de Rossilho, est presque totalement absent: c'est la vivacité du récit qui en empêche l'emploi.

8. - La poésie lyrique des troubadours connait, en plus, une autre opposition marquée par les deux formes du prétérit: le passé simple rend une action ponctuelle, c'est-à-dire, précisée, détérminée en ce qui concerne les données temporelles, unie, synthétisée et concentrée en un point; le passé composé, par contre, une action non ponctuelle, vaguement précisée quant a la durée, l'action pouvant parfois être considérée comme durative, ou comme itérative, mais jamais synthétisée dans un point.

Etant donné que les deux valeurs fondamentales des deux formes du prétérit, simple et composé, sont le parfait, l'état dans le présent, et l'aoriste, l'action dans le passé, ce qui apparaît aussi bien et même mieux sans le contexte, il va de soi que le contexte, sauf pour des verbes à valeur essentiellèment durative, devient décisif pour attribuer à l'une des deux formes verbales les valeurs respectives d' action ponctuelle et d' action non ponctuelle. On trouve:

Mout ai estat coindes e gais

Guilhem, comte de Peitieu, Pos de chantar m'es pres talens, 29

Toz mos amics prec, a la mort, que il vengan tuit e m'onren fort, qu'eu ai avut joi e deport

loing e pres et en mon aizi

$$
\text { ib., } 37-40
$$

et de nombreux passage avec les verbes être et avoir. Le contexte est indispensable, pour constater la valeur avec d'autres verbes, par ex.:

${ }^{3} \mathrm{Cfr}$. ce que dit Ch. Bally dans sa Linguistique générale et linguistique française, 2. éd., Berne, 1944, par. 585, note: - . . cette victoire (du fassé composé) a renforcé la tendance statique du français. Un récit en langue parlée: „Je me suis levé à cinq heures, j'ai pris le train de 6 heures 50 et suis arrivé à Genève dans l'aprèsmidir présente les événements comme une série de faits accomplis; de là, bien souvent, le manque de vie qui caractérise la narration en language courant. 
Anat ai cum cauz'enversa

lonc temps, sercan vals e tertres

Raimbaut d'Aurenga,

Er resplan la flors enversa, 33-4

doncs midonz per que"m refuda,

pois sap que tánt l'ai volguda

Bertran de Born,

Dompna, puois de mi no'us cal, 69-70

Mielhs-de-dona, que fugit ai dos ans, ${ }^{4}$

er torn a vos doloiros e plorans

Richart de Berbezilh, Atressi cum l'orifans, 50-1

Fait a mainta jonta

si que l'ost fai partir;

pois ponh

tant que 1 carros desjonh

Reimbaut de Vaquieras, ${ }^{5}$

Truan, mala guerra, 128-31

9. - Il n'en est pas autrement pour le provençal qu'écrivent les troubadours italiens.

Nous pouvons constater, en effet, qu'ils expriment les deux valeurs dans. lopposition aoriste : parfait en se servant des deux formes du prétérit:

Prions sospirs e loncs cossirs d'esmai

$\mathrm{m}^{\prime} a$ mes al cor la bella

Rambertis de Buvalel, Al cor m'estai l'amoros desiriers, 17-8

granz esfortz fi quant me loignei de vos

$$
\text { ib., } 44
$$

Mout se fenh prims e savis; per sos sens es tals, qu'a son tort l'a partit de si'l coms proensals, e l'autre coms no'l vol

Sordel,

Sol que m'afi ab armas tos temps del sirventes, 9-11

Ar l'ai tocat al viu, car sap qu'ieu dic, cantan, ver de sos caitiviers

$$
\text { ib., } 38-9
$$

${ }^{4}$ dos ans: la détermination temporelle est à prendre comme un terme plutôt. vague.

${ }^{5}$ Comme il l'a déjà montré Renzo Lo Cascio, L'itinerario di guerra di Rambaldo di Vaqueiras in Sicilia, dans le Bollettino del Centro di studi siciliani, V, (1957), Palermo, Raimbaut de Vaqueiras fait une distinction rigoureuse entre les notions d'action ponctuelle et action non ponctuelle. Cfr. une laisse en -0 : „Et ai estat... e n'ai a vostre pro fag maint assaut et ars mainta maizo ... Et a Messina vos cobri del blizo: En batalha vos vinc en tal sazo Que us ferion pel pietz e pel mento... Senher Marques, ja no'm diretz de no, Que vertatz es, e vos sabetz ben quo Me tinc ab vos a ley de vassalh bo, Quant assalhis a Cart... et ieu torney al maior obs. que us fo.ı 
Ar vos ai dig cum renha ni de que's sap formir

ib., 48

Plancher vuelh en Blacatz en aquest leugier so,

ab cor trist e marrit; et ai en be razo,

qu'en luy ai mescabat senhor et amic bo,

e quar tug l'ayp valent en sa mort perdut so

$$
\text { Id., Planher vuelh en Blacatz, 1-4 }
$$

Qu'eu er aitals com selva de poder anz que meu oill m'aguesson falsamen trait per leis, qe'm conquistet rizen.

Lanfrancs Cigala, Non sai' si'm chant, pero eu n'ai voler, 21-3

mas trait m'an li meu oill desleial, con trai lo bosc lo fustz de la destral

ib., $29-30$

Bona dompna, vostr'avinenz respos m'es tan plazens e m'a faig tan joios, c'oblidat n'ai mon enoi e mon mal

$$
\text { ib., } 71-3
$$

Les deux formes du prétérit peuvent servir aussi à rendre les valeurs respectives d'action ponctuelle et d'action non ponctuelle; on le voit bien dans un passage de Sordel:

Sordels diz mal de mi, e far no lo'm deuria, qu'ieu l'ai tengut (e tenh) car e onrat tot dia: donei 1 i fol, molin e autra manentia, e donei li mollier aital com el volia

Toz hom me van disen en esta maladia, 7-10

10. - Les oeuvres des troubadours ne connaissent pas, semble-t-il, le passé composé avec la valeur d'une action tout récemment passé. Certes, dans les conversations, le passé composé est la forme prédominante; mais, celles-ci sont peu nombreuses, et encore la forme verbale pourrait être interprétée comme un parfait ou bien comme une action non ponctuelle.

D'autre part, même dans les conversations, nous trouvons parfois des passés simples, comme dans le passage déjà cité: Morgues, car venguis? Ici, il est certain, que l'action passée a eu lieu dans un passé immédiat, récent.

L'opposition action éloignée : action récente, tellement importante dans les phases postérieures des langues romanes, et la seule que les grammaires normatives, toutes ou presque toutes, dans quelques langues romanes constatent et conseillent d'observer, n'existe donc pas dans la langue des troubadours.

11. - Les exemples cités ${ }^{6}$ nous inclinent à penser - quoique une conclusion fondée sur l'examen des ceuvres en vers puisse toujours être mise en doute, parce qu'on ne saurait jamais complètement éliminer l'éventuelle in- 
fluence du rythme et même de la rime - que les troubadours provençaux se servent des deux formes du prétérit, mais avec une valeur spéciale pour chacune. Le passé simple exprime l'action verbale pure, qui est en même temps unie, synthétisée en un point; ainsi, il se trouve en opposition avec le passé composé qui, lui, exprime un état dans le présent comme sa valeur originaire, primaire; mais le passé simple peut être, en tant qu'action ponctuelle, aussi en opposition avec le passé composé quand cette forme verbale exprime une action non ponctuelle. Il s'agit ici, pour le passé compiosé, d'une valeur secondaire, rendue claire surtout par le contexte. Les troubadours provençaux ne connaissent que ces deux oppositions; ils ne se servent pas des deux formes du prétérit pour distinguer entre une action récente et une action éloignée.

\section{POVZETEK}

\section{Enostavni in sestavljeni preterit. $v$ jeziku trubadurjev}

Znano je, da se $v$ stari francoščini in tako tudi $v$ provansalskih tekstih dokaj svobodno uporabljata enostavni in sestavljeni preterit, dostikrat pa namesto teh dveh časov tudi prezent.

Avtor misli, da ima $v$ veliki večini primerov sestavljeni preterit še svojo originalno vrednost, namreč vrednost perfekta, stanja $v$ sedanjosti, ki je rezultat dejanja $\mathrm{v}$ preteklosti. S tako vrednostjo stoji $\mathrm{v}$ opoziciji $\mathrm{z}$ enostavnim preteritom, ki izraža čisto glagolsko dejanje in ima potemtakem vrednost aorista.

Poleg tega lahko stojita obe obliki preterita $\mathrm{v}$ opoziciji, kadar izražata ena enovito dejanje $\mathrm{v}$ preteklosti, druga pa neenovito, včasih durativno, včasih iterativno. Ta opozicija, nastala iz prve, je sekundarna in jo moremo dodobra ugotoviti samo iz konteksta.

Vzrok za pogosto rabo sestavljenega preterita pri trubadurjih, zlasti $\vee$ epski pripovedi, je po avtorju stilne narave: serija enostavnih preteritov zagotavlja miren tok pripovedi in sicer hitro, ampak togo zaporedje dogodkov; analitični preterit $\mathbf{z}$ vrednostjo perfekta pa zahteva za seboj, ker pač izraža stanje v prezentu, kratko cezuro in zaradi nje razbija enakomerno pripoved ter tako ostreje izpostavi glagolsko dejanje, ki sledi.

${ }^{6}$ Nous nous sommes servis de textes publiés par Carl Appel, Provenzalische Chrestomathie, 6ème éd., Leipzig, 1930, et par Bartsch-Koschwitz, Chrestomathie provençale, 6ème éd., Marburg, 1904.

Les troubadours italiens, par contre, sont cités d'après Le origini: testi latini, italiani, provenzali e franco-italiani, oeuvre apparue comme vol. 1 de la collection La letteratura italiana. Storia e testi, Milano-Napoli, 1956. 